\title{
Detectability of GRB blast wave neutrinos in IceCube
}

\section{Lili Yang*}

Laboratory for Astroparticle Physics, University of Nova Gorica, 5000 Nova Gorica, Slovenia E-mail: lili.yang@ung.si

\section{Soebur Razzaque}

Dept. of Physics, University of Johannesburg, PO Box 524, Auckland Park 2006, South Africa

E-mail: srazzaque@uj.ac.za

\begin{abstract}
Accelerated ultrahigh-energy cosmic rays (UHECRs) in long-lived gamma-ray burst (GRB) blast waves are expected to interact with X-ray to optical-infrared photons of GRB afterglow to produce $\mathrm{PeV}-\mathrm{EeV}$ neutrinos. These long-lived neutrino fluxes can last for a time scale of days to years, in contrast to the prompt neutrino fluxes under the internal shocks model with a time scale of seconds to minutes and which has been constrained by recent IceCube GRB search. We calculate the expected neutrino events in IceCube in the PeV-EeV range from the blast wave of longduration GRBs, both for individual nearby GRBs and for the diffuse flux. We show that EeV neutrinos from the blast wave of an individual GRB can be detected with long-term monitoring by a future high-energy extension of IceCube for redshift up to $\mathrm{z} \sim 0.5$. We also show that with 5 years operation IceCube will be able to detect the diffuse GRB blastwave neutrino flux and distinguish it from the cosmogenic GZK neutrino flux if the UHECRs are heavy nuclei.
\end{abstract}

The 34th International Cosmic Ray Conference,

30 July- 6 August, 2015

The Hague, The Netherlands

* Speaker. 


\section{Introduction}

Gamma-ray bursts (GRBs) are the most powerful explosions in space, with a total released energy up to $10^{54}$ ergs over time scales of a few milliseconds to several hours. Long-duration GRBs with time duration $>2 \mathrm{~s}$ are thought to be caused by core collapse of massive $\left(\mathrm{m} \geq 30 M_{\odot}\right)$ stars to black holes $[1,2]$ with subsequent emission of relativistic jets of particles. They have been suggested as promising candidate sources of ultra-high energy cosmic rays (UHECRs) above $\sim 10^{18} \mathrm{eV}[3,4]$, which are accelerated in both internal and external shocks. The co-accelerated electrons in the same regions emit synchrotron radiations dominantly in $\mathrm{keV}-\mathrm{MeV}$ gamma-rays in case of internal shocks and in gamma-rays, X-rays, Optical and radio in case of external shocks. The latter case is called afterglow emission [5, 6], which have been successfully described by a number of satellite observations such as the Swift [7], Large Area Telescope (LAT) [8] and Gamma-ray Burst Monitor (GBM) [9]; both onboard the Fermi Gamma Ray Space Telescope .

In case of external forward shocks, GRB jet drives cosmic rays (assumed dominantly protons) from circumburst medium to form a blastwave. The interactions of accelerated protons in the shocks and electron-synchrotron photons lead to pion and kaon production via $p+\gamma \rightarrow \pi^{ \pm}, K^{ \pm}$, and hence PeV-EeV neutrinos can be produced efficiently by decays of $\pi^{ \pm}, K^{ \pm}$, such as $\pi^{+} / K^{+} \rightarrow$ $\mu^{+}+v_{\mu} \rightarrow e^{+}+v_{e}+\bar{v}_{\mu}+v_{\mu}$. This $v$ flux can last for quite a long time depending on the initial jet bulk Lorentz factor. Detection of blast wave neutrinos can reveal the baryonic component of GRB jets, confirm the GRBs as the source of UHECRs and help to understand GRB progenitors.

Recently, the IceCube Neutrino Observatory has reported the most stringent limit on the prompt GRB neutrino flux [10,11,12] at energies above $\sim 1 \mathrm{TeV}$ with their four-year data [13, 14]. More interestingly, IceCube helped to discover the rare and unusual Type IIn SN PTF12csy by chance with the neutrino doublet alert [15]. This coincidental detection shows the capacity to reveal transient high energy neutrino sources with neutrino telescopes. The ANTARES neutrino detector has also derived a prompt GRB neutrino flux limit with the data from 2007 to 2011 [16], which is one order of magnitude weaker than the limit from IceCube. In the ultra-high energy range $\left(E \geq 10^{15} \mathrm{eV}\right.$ ), Askaryan Radio Array (ARA) [17, 18], RICE [19] and the surface detectors (SD) of the Pierre Auger Observatory [20] are also capable to place the limits on GRB neutrino flux.

To date, no $v$ correlated to GRB has been observed. However with such obtained neutrino flux limits, people can make more stringent constraints on the fireball model, such as the emission radius or the bulk Lorentz factor. On the other hand, even if GRBs are not the significant sources of UHECRs, the future observation of these neutrinos can still probe the baryonic fraction and magnetic filed etc. In this work, we calculate both the individual $R_{i}(z)$ and the diffuse neutrino flux of GRB blast wave and provide the expected number of events in IceCube and the Pierre Auger Observatory for both cases.

\section{GRB Blast Wave Neutrino Fluence}

The blast wave neutrino flux models have been studied with different scenarios, such as an adiabatic/radiative blast wave in constant density/power law density/realistic density of external medium $[21,22,23]$. In this work, we only take the simplest evolution scenario of adiabatic blast 
wave propagating into an Inter-Steller Medium (ISM) of constant density as the case, for detailed information, see Ref. [22, 24].

The GRB ejecta from the central engine combine and drive a blast wave, which slows down by interacting with surrounding medium. After a certain time $t_{d e c}$, when the total energy in the blast wave is equal to kinetic energy of the ejecta, the blast wave decelerates in a self-similar fashion [25]. This time scale is given by

$$
t_{d e c}=33.3(1+z)\left(\frac{n}{1 \mathrm{~cm}^{3}}\right)^{-1 / 3}\left(\frac{E_{k}}{10^{55} \mathrm{erg}}\right)^{1 / 8}\left(\frac{\Gamma_{0}}{316}\right)^{-8 / 3} \mathrm{~s},
$$

where $n$ is the particle number per cubic centimeter of ISM, the initial bulk Lorentz factor $\Gamma_{0}$ is assumed to be $10^{2.5}$ and an isotropic-equivalent kinetic energy $E_{k}=10^{55} \mathrm{erg}$. The bulk Lorentz factor of an adiabatic blast wave evolving in an uniform density ISM can be expressed as,

$$
\Gamma(t)=124(1+z)^{3 / 8}\left(\frac{n}{1 c m^{3}}\right)^{-1 / 3}\left(\frac{E_{k}}{10^{55} \text { erg }}\right)^{1 / 3}\left(\frac{t}{100}\right)^{-3 / 8} .
$$

In the external forward shocks, cosmic-ray protons can be accelerated to a maximum energy of $\sim 10^{20} \mathrm{eV}$, and its spectrum is assumed to be $E_{p}^{-2}$, where $E_{p}$ is the proton energy. In the standard fireball model, the shock-accelerated electron spectrum is supposed to have three breaks due to three characteristic Lorentz factors: (1) minimum cutoff $\gamma_{m}$, depending on the shock velocities; (2) cooling $\gamma_{c}$ when electrons cools down in a blast wave expansion time; and (3) saturation $\gamma_{s}$ or maximum. Therefore, the resulting afterglow synchrotron spectrum follows a broken power law [6]. The corresponding optical depth $\tau_{p \gamma}$ for $p \gamma$ interactions is a function of cosmic-ray proton energy, which has the same shape of the characteristic synchrotron photon spectra with two or more breaks. See Ref. [22] for a detailed description.

With the proton spectrum and $\mathrm{p} \gamma$ opacity, charged pion flux can be calculated, and therefore the neutrino fluxes from pion decay can be obtained as in Ref. [24]. The blast wave neutrino flux can last for a quite long time $\sim$ one year, until the blast wave becomes non-relativistic with a Lorentz factor $\Gamma \sim 1$. Figure 3 shows single flavor neutrino fluence for kinetic energy $E_{k}=10^{55} \mathrm{ergs}$ and different redshift.

\section{Diffuse GRB Blast Wave Neutrino Flux}

To calculate the diffuse GRB blast wave neutrino flux, the population of GRBs is needed as an input. Based on the data sample with redshift information from the Swift satellite which is capable of detecting GRBs from prompt to afterglow, the fitted distribution of long GRBs density $\rho(z, L)$ has been obtained recently [26]. It is characterized by two properties, $\rho(z, L)=\phi(L) R(z)$; the luminosity function $\phi(L)$, defined as the fraction of GRBs with a given luminosity and the cosmic GRB rate $R(z)$, defined as the number of GRBs per unit comoving volume and time at a given redshift. The resulted differential luminosity function is given in terms of broken power law as

$$
\phi(L)=\left\{\begin{array}{l}
\left(\frac{L}{L_{\star}}\right)^{-a} ; L<L_{b} \\
\left(\frac{L}{L_{\star}}\right)^{-b} ; L \geq L_{b},
\end{array}\right.
$$




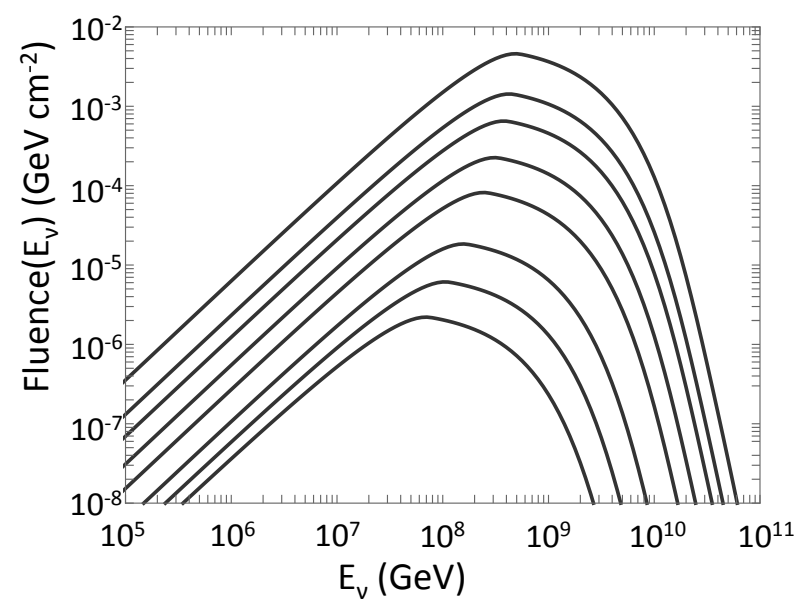

Figure 1: Neutrino fluence from GRB blastwaves in an ISM of uniform density of particles $1 \mathrm{~cm}^{-3}$ with isotropic-equivalent kinetic energy $E_{k}=10^{55} \mathrm{erg}$ at redshift of $0.3,0.5,0.7,1.1,1.7,3.3,5.5,9.0$ from top to bottom curves.

where $a=0.17, b=1.44$ and $L_{b}=10^{52.5} \mathrm{erg} / \mathrm{s}$. The corresponding GRB rate per comoving volume and time in the interval $z$ to $z+d z$ is,

$$
R_{i}(z)=R_{0}\left\{\begin{array}{lr}
(1+z)^{a} ; \quad & z<z_{b} \\
(1+z)^{-b} N ; & z \geq z_{b},
\end{array}\right.
$$

where the local GRB rate density $R_{0}=1.25 \mathrm{Gpc}^{-3} \mathrm{yr}^{-1}$, break redshift $z_{b}=3.11$, the indices $a=2.07, b=1.36$, and the normalization factor $N=127.49$. Therefore, the differential comoving rate of long GRBs at a given redshift $z$ can be expressed as,

$$
R(z)=\frac{R_{i}(z)}{1+z} \frac{d V(z)}{d z}
$$

with comoving volume element $d V / d z$ and the cosmological time dilation factor $(1+z)^{-1}$. The cumulative GRB number per unit time interval can be calculated as $R_{c}\left(z^{\prime}\right)=\int_{0}^{z^{\prime}} \int_{L_{1}}^{L_{2}} \rho(z, L)$. For instance, within redshift $z^{\prime}=0.2$, the rate of GRBs is $\sim 10$ per year with luminosity $2.5 \times 10^{49}-$ $2.5 \times 10^{50} \mathrm{erg}$.

The diffuse neutrino flux can be obtained by integrating individual GRB fluence $F_{V}\left(E_{v}, z\right)$ over luminosity and redshift distribution of GRB density $\rho(z, L)$ as,

$$
J_{v}(E)=\frac{1}{16 \pi^{2}} \int_{z_{1}}^{z_{2}} \int_{L_{1}}^{L_{2}} \rho(z, L) F_{v}\left(E_{v}, z\right) d L d z
$$

where the upper and lower redshift limits are $z_{1}=0.1, z_{2}=9.0$ based on the GRB observations. We assume the relation of kinetic energy in blast wave and gamma ray luminosity as $E_{k}=f_{b} \eta_{\gamma} t L$ [27], with a baryon loading factor $f_{b}=20$, typical time duration of long GRBs $\sim 10 \mathrm{~s}$ and efficiency in converting the kinetic energy into gamma rays $\eta_{\gamma}=0.2$. With isotropic equivalent kinetic energy $E_{k}$ in the $10^{51}-10^{55} \mathrm{ergs}$, the range of $\gamma$-ray luminosity can be set as $L_{1}=2.5 \times 10^{49} \mathrm{erg} / \mathrm{s}$ and 


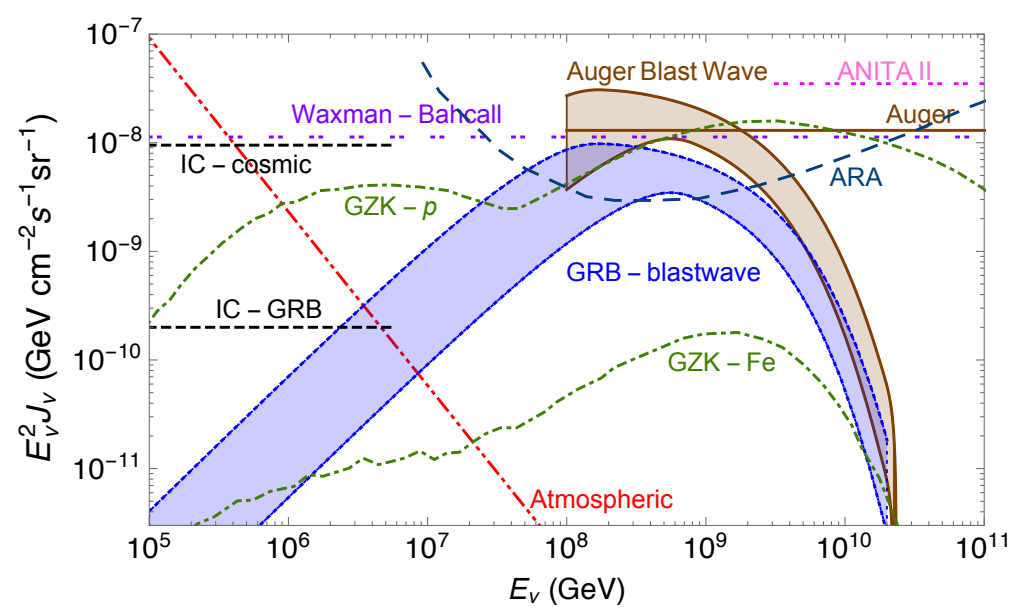

Figure 2: The diffuse $v_{\mu}+\bar{v}_{\mu}$ fluxes from GRB blast wave (shaded blue) in constant particle density of $1 \mathrm{~cm}^{-3}$ (lower blue dotted) and $10 \mathrm{~cm}^{-3}$ (upper blue dotted), averaged atmospheric neutrino flux (red dotdash-dot)[29], GZK neutrino fluxes from proton (upper green dot-dash) and iron (lower green dot-dashed) dominant primaries [30] are shown and Waxman-Bahcall limit [33] are also shown. The diffuse neutrino flux limits from IceCube (black short-dash) [31], ARA (dark green dashed) [17], ANITA-II (magenta dot-dot) [32] the Pierre Auger Observatory (brown solid) [20] and the most recent IceCube GRB neutrino flux limit (upper black short-dash) and Auger GRB limit of this model (brown shaded) are displayed here.

$L_{2}=2.5 \times 10^{53} \mathrm{erg} / \mathrm{s}$. The particle density $n_{0}$ is assumed to be $1 \mathrm{~cm}^{-3}$ as in a typical disc ISM and $10 \mathrm{~cm}^{-3}$ in a dense ISM. Figure 2 shows the computed diffuse single flavor GRB blast wave neutrino flux after oscillation (blue shaded region) with uniform particle density of ISM $n_{0}=1,10$ $\mathrm{cm}^{-3}$ (lower and upper borders of the shaded region respectively). Within the period of January 2004 to June 2013, there is no candidate found in the Pierre Auger Observatory neutrino search [20]. With the information of neutrino exposure of the surface detector as Fig. 3 of Ref. [20], an upper limit to the diffuse blast wave neutrino flux can be computed based on the blast wave neutrino flux model of this work. We place $90 \%$ confidence level upper limits on the flux model in the Auger GRB neutrino search, assuming the differential single flavor blast wave neutrino flux is $d N / d E=k J_{v}(E)$, where $\mathrm{k}$ is the normalization factor. The upper limit on the value of $k$ can be computed, according to the Feldman-Cousins approach [28],

$$
k=\frac{N_{90}}{\int_{E_{v}} k J_{v}\left(E_{v}\right) d E_{v}}
$$

where $N_{90}=2.44$ is the experimental sensitivity for $90 \%$ confidence level with zero true signal and zero background signal, and the interval of energy integral is $1.0 \times 10^{8}-2.5 \times 10^{10} \mathrm{GeV}$, where $90 \%$ of events is expected [20]. The calculated Auger GRB limits of this model are shown as brown shaded region with solid curves in Fig. 2, with $1 \mathrm{~cm}^{-3}$ (upper curve) and $10 \mathrm{~cm}^{-3}$ (lower curve) respectively. In the same figure, the background limits of averaged atmospheric neutrino [29] and GZK neutrino from proton and iron primaries in [30] are displayed. The recent diffuse neutrino flux limits from IceCube labeled as "IC-cosmic" [31], ANITA-II [32], ARA expected 3 year sensitivity [17] and the Pierre Auger Observatory [20], and IceCube GRB neutrino limit [14], together with Waxman-Bahcall limits [33] are plotted as well. 
Table 1: The combined 3 flavor neutrino events expected in IceCube from an individual GRB at redshift 0.2 with uniform particle density of $1 \mathrm{~cm}^{-3}$ and $10 \mathrm{~cm}^{-3}$ and the corresponding background neutrino events from atmosphere, diffuse GZK $p$-dominated and Fe-dominated in three energy bins respectively.

\begin{tabular}{|c|c|c|c|}
\hline $\log _{10} \mathrm{E}(\mathrm{GeV})$ & $5.5-7$ & $7-8.5$ & $8.5-10$ \\
\hline $\mathrm{GRB} 1 / \mathrm{cm}^{-3}$ & $2.00 \times 10^{-3}$ & $4.32 \times 10^{-2}$ & $5.26 \times 10^{-2}$ \\
\hline $\mathrm{GRB} 10 / \mathrm{cm}^{-3}$ & $2.57 \times 10^{-2}$ & 0.26 & 0.11 \\
\hline Atm. & $1.47 \times 10^{-4}$ & $6.36 \times 10^{-6}$ & $1.20 \times 10^{-8}$ \\
\hline$G Z K_{F e}$ & $1.19 \times 10^{-5}$ & $2.27 \times 10^{-5}$ & $3.52 \times 10^{-5}$ \\
\hline$G Z K_{p}$ & $4.12 \times 10^{-3}$ & $2.54 \times 10^{-3}$ & $3.33 \times 10^{-3}$ \\
\hline
\end{tabular}

\section{Expected Neutrino Events}

Individual GRBs are treated as point sources, so the expected number of neutrino events from single nearby GRB can be calculated with $N_{\mathrm{obs}, \alpha}=\int_{E_{v 1}}^{E_{v 2}} d E_{v} A_{\mathrm{obs}, \alpha}\left(E_{v}\right) F_{v_{\alpha}}\left(E_{v}\right)$, here $\alpha$ refers to the flavors of neutrinos, and the subscript "obs" represents various detectors, here as IceCube and the Pierre Auger Observatory, and $F_{v_{\alpha}}$ is the fluence of individual GRBs. In IceCube, the secondary neutrinos of GZK effects and atmospheric neutrinos are the main backgrounds, their number of events for point sources searching is calculated as,

$$
N_{\mathrm{bkg}, \alpha}=2 \pi \frac{\delta \theta^{2}}{2} T \int_{E_{v 1}}^{E_{v 2}} d E_{v} A_{\mathrm{ic}, \alpha}\left(E_{v}\right) F_{v_{\alpha}}\left(E_{v}\right)
$$

where $\delta \theta$ is the angular resolution of IceCube, $1^{\circ}$ and $10^{\circ}$ for track and cascade events separately and $T$ is the time duration of neutrino flux from blast wave. The expected number of events for a single GRB at redshift 0.2 with constant particle density and background events can be found in the Table 1. To get the number of diffuse blast wave neutrino events or background events, we use the equation $N_{\mathrm{obs}, \alpha}=\Omega T \int_{E_{v 1}}^{E_{v 2}} d E_{v} A_{\mathrm{obs}, \alpha}\left(E_{v}\right) J_{\alpha}\left(E_{v}\right)$, where $\Omega$ is the observing solid angle, and $T$ is the running time. The number distribution of muon neutrino events in IceCube is shown in Fig. 3. We can see that the backgrounds of GZK neutrinos from proton primary are dominant most of the energy range. However, if the UHECRs are heavy nuclei, IceCube will be able to distinguish it from the cosmogenic GZK neutrino flux easily. For the SD of Auger, we estimate that 1.13 diffuse blast wave neutrino events of all three flavors will be observed during $\sim 6.4$ years operating time with energy of $10^{7}-10^{11} \mathrm{GeV}$ for highly-inclined down-going and earth-skimming channels.

\section{Discussion}

In this work, based on the model of adiabatic blast wave evolving in uniform density ISM, we have computed the neutrino fluence of individual GRB at various redshifts and as well the diffuse blast wave neutrino flux, thanks to the recent fitted long GRB density rate. Our most optimistic diffuse neutrino flux is higher than the ARA three-year sensitivity [17] in the $0.03-2 \mathrm{EeV}$ range and the flux peak is slightly lower than Waxman-Bahcall limit [33] and Auger diffuse neutrino limit [20]. Based on the same model, we have placed the diffuse GRB blast wave neutrino limits for the $\mathrm{SD}$ of Auger, which is lower than the Waxman-Bahcall limit in some energy range. 


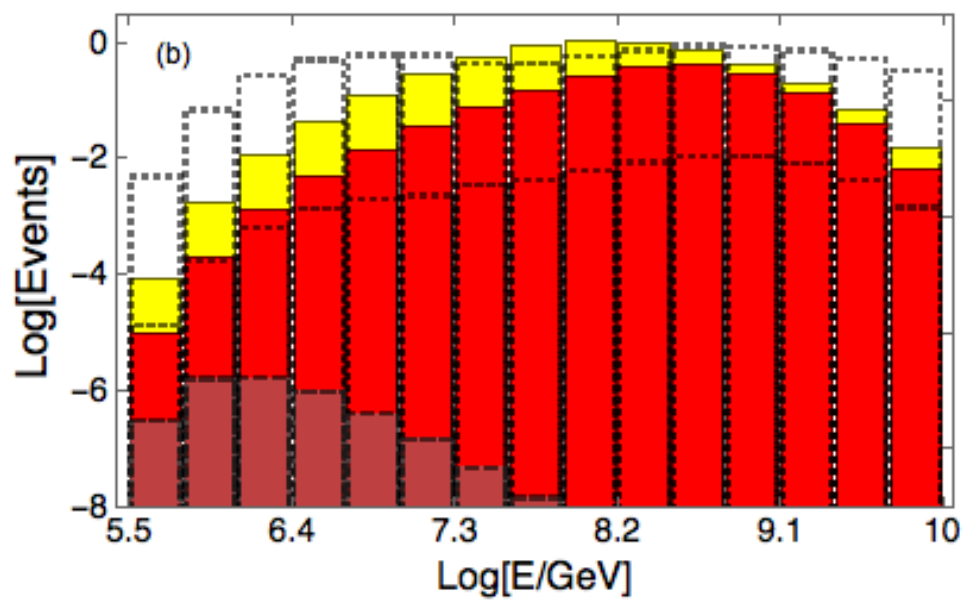

Figure 3: Expected number distribution of diffuse $v_{\mu}+\bar{v}_{\mu}$ events in IceCube from GRB blast wave in constant particle density ISM of $1 \mathrm{~cm}^{-3}$ (red, solid-edge, lower) and $10 \mathrm{~cm}^{-3}$ (yellow, solid-edge, higher), atmosphere (shaded, dashed edge), GZK-p dominated (transparent, dotted edge, higher) and GZK-Fe dominated (transparent, dotted edge, lower) in 10 year running time.

We have also calculated the expected number of events in IceCube and the Pierre Auger Observatory with the individual neutrino fluence and diffuse flux. If the model is realistic, within 5-year operation, IceCube can observe up to 6 neutrinos of all three flavors from diffuse GRB blast wave flux. In the case that UHECRs primaries are proton dominant, the neutrino observation will take a longer time. With the expansion of IceCube detector, IceCube-Gen2 [34], whose size is 10 times of the current IceCube, it will be possible to observe neutrinos from single GRBs nearby. For instance, IceCube-Gen2 can detect up to 4 events from an energetic GRB at $\mathrm{z} \sim 0.2$.

\section{References}

[1] A. MacFadyen and S. E. Woosley, Astrophys. J. 524, 262 (1999) [astro-ph/9810274].

[2] S. Woosley and A. Heger, Astrophys. J. 637, 914 (2006) [astro-ph/0508175].

[3] E. Waxman, Phys. Rev. Lett. 75, 386 (1995) [astro-ph/9505082].

[4] M. Vietri, Astrophys. J. 453, 883 (1995) [astro-ph/9506081].

[5] P. Meszaros and M. J. Rees, Astrophys. J. 476, 232 (1997) [astro-ph/9606043].

[6] R. Sari, T. Piran and R. Narayan, Astrophys. J. 497, L17 (1998) [astro-ph/9712005].

[7] T. Sakamoto, S. D. Barthelmy, W. H. Baumgartner, J. R. Cummings, E. E. Fenimore, N. Gehrels, H. A. Krimm and C. B. Markwardt et al., Astrophys. J. Suppl. 195, 2 (2011) [arXiv:1104.4689 [astro-ph.HE]].

[8] M. Ackermann et al. [Fermi-LAT Collaboration], Astrophys. J. (submitted) arXiv:1303.2908 [astro-ph.HE].

[9] A. von Kienlin, C. A. Meegan, W. S. Paciesas, P. N. Bhat, E. Bissaldi, M. S. Briggs, J. M. Burgess and D. Byrne et al., Astrophys. J. Suppl. 211, 13 (2014) [arXiv:1401.5080 [astro-ph.HE]]. 
[10] E. Waxman and J. N. Bahcall, Phys. Rev. Lett. 78, 2292 (1997) [astro-ph/9701231].

[11] D. Guetta, D. Hooper, J. Alvarez-Muniz, F. Halzen and E. Reuveni, Astropart. Phys. 20, 429 (2004) [astro-ph/0302524].

[12] S. Hummer, P. Baerwald and W. Winter, Phys. Rev. Lett. 108, 231101 (2012) [arXiv:1112.1076 [astro-ph.HE]].

[13] R. Abbasi et al. [IceCube Collaboration], Nature 484, 351 (2012) [arXiv:1204.4219 [astro-ph.HE]].

[14] M. G. Aartsen et al. [IceCube Collaboration], Astrophys. J. 805, no. 1, L5 (2015) [arXiv:1412.6510 [astro-ph.HE]].

[15] M. G. Aartsen et al. [IceCube and PTF and Swift and Pan-STARRS1 Science Consortium Collaborations], arXiv:1506.03115 [astro-ph.HE].

[16] S. Adrian-Martinez et al. [ANTARES Collaboration], Astron. Astrophys. 559, A9 (2013) [arXiv:1307.0304 [astro-ph.HE]].

[17] P. Allison, J. Auffenberg, R. Bard, J. J. Beatty, D. Z. Besson, S. Boser, C. Chen and P. Chen et al., Astropart. Phys. 35, 457 (2012) [arXiv:1105.2854 [astro-ph.IM]].

[18] P. Allison, J. Auffenberg, R. Bard, J. J. Beatty, D. Z. Besson, C. Bora, C. C. Chen and P. Chen et al., arXiv:1507.00100 [astro-ph.HE].

[19] S. Razzaque, J. A. Adams, P. Harris and D. Besson, Astropart. Phys. 26, 367 (2007) [astro-ph/0605480].

[20] A. Aab et al. [Pierre Auger Collaboration], Phys. Rev. D 91, no. 9, 092008 (2015) [arXiv:1504.05397 [astro-ph.HE]].

[21] C. D. Dermer, Astrophys. J. 574, 65 (2002) [astro-ph/0005440].

[22] S. Razzaque, Phys. Rev. D 88, no. 10, 103003 (2013) [arXiv:1307.7596 [astro-ph.HE]].

[23] P. Mimica and D. Giannios, Mon. Not. Roy. Astron. Soc. 418, 583 (2011) [arXiv:1106.1903 [astro-ph.HE]].

[24] S. Razzaque and L. Yang, Phys. Rev. D 91, 043003 (2015) [arXiv:1411.7491 [astro-ph.HE]].

[25] R. D. Blandford and C. F. McKee, Phys. Fluids 19, 1130 (1976).

[26] D. Wanderman and T. Piran, Mon. Not. Roy. Astron. Soc. 406, 1944 (2010) [arXiv:0912.0709 [astro-ph.HE]].

[27] D. A. Frail, S. R. Kulkarni, R. Sari, S. G. Djorgovski, J. S. Bloom, T. J. Galama, D. E. Reichart and E. Berger et al., Astrophys. J. 562, L55 (2001) [astro-ph/0102282].

[28] G. J. Feldman and R. D. Cousins, Phys. Rev. D 57, 3873 (1998) [physics/9711021 [physics.data-an]].

[29] M. Honda, T. Kajita, K. Kasahara, S. Midorikawa and T. Sanuki, Phys. Rev. D 75, 043006 (2007) [astro-ph/0611418].

[30] K. Kotera, D. Allard and A. V. Olinto, JCAP 1010, 013 (2010) [arXiv:1009.1382 [astro-ph.HE]].

[31] M. G. Aartsen et al. [IceCube Collaboration], Science 342, 1242856 (2013) [arXiv:1311.5238 [astro-ph.HE]].

[32] P. W. Gorham et al. [ANITA Collaboration], Phys. Rev. D 85, 049901 (2012) [arXiv:1011.5004 [astro-ph.HE], arXiv:1003.2961 [astro-ph.HE]].

[33] E. Waxman and J. N. Bahcall, Phys. Rev. D 59, 023002 (1999) [hep-ph/9807282].

[34] M. G. Aartsen et al. [IceCube Collaboration], arXiv:1412.5106 [astro-ph.HE]. 